\title{
RESEARCH HIGHLIGHT Opioid dose regimen shapes mesolimbic adaptations
}

\author{
Catherine M. Cahill ${ }^{1}$ \\ Neuropsychopharmacology (2020) 45:1777-1778; https://doi.org/10.1038/s41386-020-0679-y
}

Prescription, diversion, and illicit use of opioid therapeutics have emerged as a major societal concern in recent years, fueling a concerted effort to identify the neural basis of opioid dependence, with the goal of developing more effective treatments for those afflicted with substance use disorders. One question that continues to be explored due to its substantial clinical implications is: to what extent does opioid dosing and pharmacokinetics (continuous versus interrupted opioid administration) influence abuse liability and modify reward circuitry? The clinical relevance goes beyond the desire to maintain analgesia while minimizing rescue medication to treat breakthrough pain. A valuable lesson in appropriate dosing was learned from Oxycontin ${ }^{\circledR}$ prescription recommendations, where those taking this opioid often exhibited pseudo-addiction due to the mis-guided marketing that the pharmacokinetic profile recommended dosing every $12 \mathrm{~h}$. In reality, the half-life of this extended release formulation was much shorter, ranging from 4.5 to $9 \mathrm{~h}$ (SPC. Oxycontin. https://www.accessdata.fda.gov/drugsatfda_docs/label/ 2015/022272s027lbl.pdf). The lack of maintaining steady state opioid plasma levels not only allowed break through pain to occur, but likely generated stress and/or mild symptoms of withdrawal. Thus, it is not unreasonable to assume that the inappropriate opioid dosing contributed to the current climate of the opioid epidemic. Preclinical research supports the idea that the lack of steady state opioid plasma levels impacts drug-seeking behavior, where drug regimens had dramatic effects on mesolimbic circuits, including dopamine-dependent reward. Yu et al. [1] determined that while both continuous versus intermittent morphine dosing produced analgesic tolerance, only the intermittent morphine dosing facilitated subsequent morphine self-administration. In addition, Lee et al. [2] recently reported that intermittent (but not continuous) opioid administration induced neuroinflammation and dysregulation of dopamine-dependent reward behaviors, which was causally related to changes in gut microbiome.

In the current issue of Neuropsychopharmacology $\odot$, Lefevre et al. [3] provide further insight to mechanisms underlying opioid-induced adaptive changes in brain regions involved in motivation, reinforcement, decision-making, and reward prediction. They report that intermittent daily morphine (Q24h) causes long-lasting psychomotor sensitization, whereas continuous morphine (via infusion pump) produced the opposite effect exhibiting locomotor tolerance. The phenomenon of locomotor sensitization requires striatal dopamine release and is often used as a proxy for assessing plasticity of reward circuitry [4]. To eliminate the possibility that opioid plasma concentrations were responsible for the different behavioral outcomes, the authors administered twice daily naloxone $(\mathrm{Q} 12 \mathrm{~h})$ to mice receiving continuous morphine to recapitulate an intermittent dosing paradigm. Interestingly, the dose-dependent locomotor sensitization effects persisted following a 10-day cessation of the initial morphine, suggesting that it produced significant synaptic plasticity in striatal circuitry. To determine if changes in basal dopamine levels could account for the differences in locomotor sensitization between the two dosing regimens, the Leferve study used an elegant fiber photometry approach. They expressed the genetically encoded dopamine sensor dLight $1.3 \mathrm{~b}$ in the nucleus accumbens and measured the fluorescent signal prior to and following different morphine dosing regimens. Spontaneous fluorescent transient events were used to assess baseline dopamine dynamics. Intermittent morphine increased the amplitude of these events, whereas continuous morphine reduced it, suggesting dynamic differences in dopamine signaling and mesolimbic circuit activity.

The interesting commonality between many of the studies that examined differences between continuous and intermittent opioid dosing is that continuous opioid exposure, that produces steady state blood and brain opioid levels, does not cause significant dysregulation of the mesolimbic dopaminergic circuit. Yet, dopamine-dependent behavior is modified when opioid exposure is interrupted, presumably by imposing a state of repeated acute withdrawal. According to Lefevre et al. [3] patterns of opioid exposure elicit differences in the transcriptome within the striatum, a key brain region involved in the genesis of substance use disorders. Transcriptional profiling, showed that intermittent, but not continuous, opioid exposure altered mRNA expression of both excitatory and inhibitory transmitter receptor subunits [3]. These changes may contribute to the synaptic plasticity within mesolimbic circuitry that play a role in addictive-like behaviors in animal models. One of the modified transcripts highlighted in the Lefevre paper was heat shock transcription factor 1 (HSF1), a DNA binding protein activated following cellular stress. Inhibitors of HSF1 have been pursued as a novel cancer treatment [5], and there is strong evidence that HSF1 function is neuroprotective and reduced in neurodegenerative disorders such as Alzheimer's disease [6]. Further studies are required to define the potential of this target in treating substance use disorders.

Is it possible that opioid dosing regimens partially explain reports that opioid use can increase the risk of depression recurrence and development of treatment-resistant depression even after controlling for pain, psychiatric disorder and opioid misuse (reviewed in [7])? Intermittent (but not continuous) opioid dosing leads to neuroinflammation [2], which can precipitate protracted abstinence syndrome and negative mood states that is thought to drive drug craving. Methadone maintenance therapy is effective because of its

\footnotetext{
${ }^{1}$ Department of Psychiatry and Biobehavioral Sciences, Shirley and Stefan Hatos Center for Neuropharmacology, Jane \& Terry Semel Institute for Neuroscience and Human Behavior, University of California Los Angeles, Los Angeles, CA, USA90095

Correspondence: Catherine M. Cahill (cmcahill@ucla.edu)
}

Received: 27 March 2020 Accepted: 3 April 2020

Published online: 29 April 2020 
long half-life, which minimizes or eliminates the euphoric effects associated with acute opioid use such as heroin or fentanyl. But what if this explanation is an oversimplification. Maybe methadone normalizes the striatal neuroplasticity and/or transcript changes associated with opioid misuse due to its ability to maintain steady state plasma levels. Indeed, methadone was shown to reduce emotional and depressive reactivity in subjects on methadone maintenance therapy [8]. Could the concept that dosing influences reward circuitry be generalizable to other drugs of abuse? Clinical trials assessing the effectiveness of methylphenidate for maintenance therapy to psychostimulant addiction show mixed results, although there was some indication that it increased retention in subjects with cocaine addiction [9], and improved depressed $\operatorname{mood}[10]$.

In conclusion, the Lefreve study, and others like it, remind us that choice of dose regimens influences outcomes, and that modeling human drug use is critical if we are to understand the underlying mechanisms that lead to substance use disorders and comorbid diseases. Further, more studies are needed to understand whether it is the stress per se (caused by repeated withdrawal that accompanies the intermittent dosing regimen), the learned association that subsequent drug use alleviates negative affect and withdrawal, or another factor such as neuroinflammation that sets up the perfect storm for addiction susceptibility.

\section{FUNDING AND DISCLOSURES}

This author was supported by the Shirley Hatos Foundation, NIHNational Institute of Drug Abuse Grant numbers R01DA041781, 1UG3TR003148-01, and 2P50 DA005010, and the Department of Defense Grant number W81XWH-15-1-0435. Nothing to disclose and I have no competing interests.

\section{ADDITIONAL INFORMATION}

Publisher's note Springer Nature remains neutral with regard to jurisdictional claims in published maps and institutional affiliations.

\section{REFERENCES}

1. Yu G, Zhang F-Q, Tang S-E, Lai M-J, Su R-B, Gong Z-H. Continuous infusion versus intermittent bolus dosing of morphine: a comparison of analgesia, tolerance, and subsequent voluntary morphine intake. J Psychiatr Res. 2014;59:161-6.

2. Lee K, Vuong HE, Nusbaum DJ, Hsiao EY, Evans CJ, Taylor AMW. The gut microbiota mediates reward and sensory responses associated with regimenselective morphine dependence. Neuropsychopharmacology. 2018;43:2606-14.

3. Lefevre EM, Pisansky MT, Toddes C, Baruffaldi F, Pravetoni M, Tian L, et al. Interruption of continuous opioid exposure exacerbates drug-evoked adaptations in the mesolimbic dopamine system. Neuropsychopharmacology (2020). https://doi.org/10.1038/s41386-020-0643-x

4. Scofield MD, Heinsbroek JA, Gipson CD, Kupchik YM, Spencer S, Smith ACW, et al. The nucleus accumbens: mechanisms of addiction across drug classes reflect the importance of glutamate homeostasis. Pharmacol Rev. 2016;68:816-71.

5. Dong B, Jaeger AM, Thiele DJ. Inhibiting heat shock factor 1 in cancer: a unique therapeutic opportunity. Trends Pharmacol Sci. 2019;40:986-1005.

6. Calderwood SK, Murshid A. Molecular chaperone accumulation in cancer and decrease in alzheimer's disease: the potential roles of HSF1. Front Neurosci 2017;11:192.

7. Evans, CJ, Cahill, CM. Neurobiology of opioid dependence in creating addiction vulnerability. F1000Research 2016;5.

8. Savvas SM, Somogyi AA, White JM. The effect of methadone on emotional reactivity. Addiction. 2012;107:388-92.

9. Miles SW, Sheridan J, Russell B, Kydd R, Wheeler A, Walters C, et al. Extendedrelease methylphenidate for treatment of amphetamine/methamphetamine dependence: a randomized, double-blind, placebo-controlled trial. Addiction. 2013;108:1279-86

10. Kerr CW, Drake J, Milch RA, Brazeau DA, Skretny JA, Brazeau GA, et al. Effects of methylphenidate on fatigue and depression: a randomized, double-blind, placebo-controlled trial. J Pain Symptom Manag. 2012;43:68-77. 